\title{
Example Use of Low-Cost System for Capturing the Kinetic Parameters of Sperm Cells in Atlantic Salmon (Sallmo sallar)
}

\author{
Jorge Parodi ${ }^{1}$, Alfredo Ramírez-Reveco ${ }^{2}$, Guillermo Guerra ${ }^{1}$ \\ ${ }^{1}$ Laboratorio de Fisiología de la Reproducción, Núcleo de Producción Alimentaria, Escuela de Medicina, \\ Veterinaria, Facultad de Recursos Naturales, Universidad Católica de Temuco, Temuco, Chile \\ ${ }^{2}$ Laboratorio de Criobiología y Análisis de Funcionalidad Espermática, Instituto de Ciencia Animal, Facultad de \\ Ciencias Veterinarias, Universidad Austral de Chile, Valdivia, Chile \\ Email: jparodi@uct.cl
}

Received 13 January 2015; accepted 28 January 2015; published 2 February 2015

Copyright (C) 2015 by authors and Scientific Research Publishing Inc.

This work is licensed under the Creative Commons Attribution International License (CC BY). http://creativecommons.org/licenses/by/4.0/

(c) (i) Open Access

\begin{abstract}
A spermiogramme is an analysis performed to assess the quality of semen. Motility parameters are primarily obtained by subjectively observing samples or by using complex systems, such as computer-assisted sperm analysis (CASA). Here, we describe an easy and low-cost analysis system for obtaining quantifiable kinetic observations using Salmo salar semen model. In this work, we observed and captured video images of both fresh and stored Atlantic salmon semen to describe the possibility of analysis using the ImageJ CASA plug-in application for the kinetic parameters obtained from the videos. The semen is exposed to "powermilt", a commercial activating solution, and the curvilinear velocity (VCL), straight line velocity (VSL), average path velocity (VAP) values are described. When the samples were activated after having been stored, differences were detected in sperm quality, using the low-cost plug-in application. However, this system was not able to detect small variations in the same recorded sample, suggesting limits in sample observation. The results indicate that it is possible to quantify the kinetic parameters of semen samples using a low-cost video system and free software.
\end{abstract}

\section{Keywords}

Fish, Semen, Motility, Low Cost

\section{Introduction}

Sperm cells are complex models, and the study of their motility is necessary in order to describe the quality of 
the samples [1], which is important in the fertilization process as faster and quantitative analysis help to identify the best sample; in recent years, computational systems have been used to measure the quality of human spermatozoa [2], and for following morphological changes [3]. Semen is manipulated in vitro at the industrial level in order to improve production. In particular, semen can be preserved, cryopreserved and then used as though fresh for fertilization or artificial insemination [4] [5]. Semen is examined for different physicochemical and cellular aspects, in particular, motility and the quality of motility [6]. In mammalian spermatozoa, observing motility is crucial for the evaluation of the sample quality and is used in various processes; the quality of a sample can be evaluated by an observer according to international scales [7] that are still used in several studies [8][10]. However, systems based on software programs (CASA, Computer-Assisted Sperm Analysis) have allowed the quantification of kinetic parameters and have enhanced the evaluation of semen quality [11] [12], yet these systems have also increased the cost of semen evaluation and reduced the availability of fast, low-cost and easy ways to conduct a preliminary study of sperm cell quality. During breeding, gametes are released into the water where the oocytes are fertilized. It is the key that sperm cells are activated and their motility started with the osmotic change in solutions [13]. This particularity of sperm cells provides time to manipulate the sample based on laboratory conditions, and the determining the kinetic values of these cells through in vitro studies allows this process to be monitored. Intrinsic gamete quality, the quantity of gametes, and the environment in which they fuse determine the success of fertilization. The kinetic properties of sperm cells are important parameters to study to ensure reproductive success and improve sperm quality; thus, observing sperm kinetics facilitates improved production [13]-[15]. Mass motility observations in fish are often performed and reported as percentages assessed by an observer, although the CASA system is developed to better assess the motility of a sample [11] [16]. Although the use of motility percentages remains valid, this process may introduce errors. Studies have indicated decreases in sperm motility in semen that were not related to the success of fertility; the absence of a relationship is explained by the fact that non-kinetic parameters were observed. Recently, several studies have demonstrated that changes in fish sperm cell kinetics are a better indicator of the quality of the semen collected and a better parameter for use in the Salmon-culture industry [12] [17] [18]. The high costs of commercial software limit their availability, as does the associated microscope necessary for the proper analysis of samples. Open source software systems have been developed, such as the CASA software for ImageJ, which is an open source plug-in application available at http://rsb.info.nih.gov/ij/plugins/casa.html that has been validated by the National Institutes of Health for the analysis of videos of particles and mammalian sperm cells, and for obtaining relevant kinetic parameters [19]. Recently, work had incorporated the use of such a CASA plug-in for analyzing the kinetics of sperm samples [20]: modifications can be made to measure the kinetics of the sample, for example, in the Steindachneridion parahybae model, the protocol of Image J was adapted for the specific sample [21]. The parameters most commonly observed by these free systems are VCL (curvilinear velocity), VAP (average path velocity) and VSL (straight-line velocity), but it is also possible to analyze other parameters, including the percentage of motile forms and the linearity of the sample [22]. This open source and commercial system enables the determination of all these parameters, thereby generating quantifiable measures of semen sample quality [19]. Thus, researchers are able to implement this analysis protocol as a cost-effective and straightforward alternative for retrieving quantitative and reproducible information in the laboratory. In this way, researchers have also begun to develop systems for the photographic analysis of sperm cell head morphology as another indicator of sperm quality [23]. In particular, this work used a Salmo salar model, because we can induce motility in laboratory condition for capture videos. This marks an interesting discussion of the applicability of freely available software and low-cost microscopy tools for this type of study and for observing the kinetics of sperm cells in several models.

\section{Materials and Methods}

\subsection{Collection and Handling of Semen Samples}

The animals and biological samples were handled in accordance with the ethics guidelines of UCT and CONICYT and approved by FONDEF project D10i1064. All of the biological materials were discarded according to UCT protocol. A sample of nine adult male Atlantic salmon (Salmo salar) was donated by BIOACUI, which is affiliated with the Universidad Católica de Temuco. Before semen extraction, the salmon were anaesthetised with a $0.015 \%$ dilution of BZ-20 (benzoate salt solution at $20 \%$ ). Semen samples were extracted through abdominal massage or "stripping" and then oxygenated and stored in airtight plastic containers, which 
were subsequently cooled at $1^{\circ} \mathrm{C}$ to $5^{\circ} \mathrm{C}$ and transported to the laboratory in cooler containing a gel pack, the transfer time from the collection center and the laboratory was approximately 2 hours. In the laboratory, semen was diluted 1:2 (semen:sperm diluent) in a solution consisting of $\mathrm{NaCl} 18.8 \mathrm{~g} / \mathrm{L}, \mathrm{CaCl}_{2} \cdot 3 \mathrm{H}_{2} \mathrm{O} 2 \mathrm{~g} / \mathrm{L}, \mathrm{KCl} 72 \mathrm{~g} / \mathrm{L}$, $\mathrm{NaH}_{2} \mathrm{PO}_{4} \cdot \mathrm{H}_{2} \mathrm{O} 4.1 \mathrm{~g} / \mathrm{L}, \mathrm{NaHCO}_{3} 1 \mathrm{~g} / \mathrm{L}, \mathrm{MgSO}_{4} \cdot 7 \mathrm{H}_{2} \mathrm{O} 2.3 \mathrm{~g} / \mathrm{L}$ and glucose $1 \mathrm{~g} / \mathrm{L}$. All procedures were performed at $4^{\circ} \mathrm{C}$ and the initial condition was 4 hours after the sample was obtained. We used a haemocyter for sperm cell count, and the sample had approximately $11 \times 10^{-9} \pm 2$ cells by $\mathrm{ml}$ before dilution, similar to values from another study [24]. The samples show an activation time of $180 \pm 10 \mathrm{~s}$ and decay at $50 \%$, similar to results from a previous study [25]. The samples were agitated daily and aerated by injecting compressed oxygen to prevent mortality of the sperm cells (which are not motile for the entire conservation period) and preserved for 20 days for periodic experiments [26].

\subsection{Observation of the Samples under a Microscope}

Slides were prepared with $10 \mu \mathrm{l}$ of semen, deposited directly over slide, and quickly mounted on a NIKON Labophot 2 optical microscope. Activation solution was used instead of coverslips, in order to maintain access to the sample. Prior to this step, all materials were maintained at $4^{\circ} \mathrm{C}$ and sperm cells were not motil. The samples utilized for observation consisted of cells with size $2 \mu \mathrm{M}$. After focusing the sample, $20 \mu \mathrm{l}$ of Powermilt (1×) activation solution [26] was applied at the edge of the drop sample to obtain interface, and activate sperm cell movement was observed. The samples were then observed at the interface of the solutions to record the activation of the spermatozoa, the border between solutions was in focus when the Powermilt was added, all of which exhibits a low-cost, easy to replicate model and in many laboratories. Time elapsed was recorded from the initiation of cell movement until all cells within the visual field had stopped moving and their kinetics were no longer observable by the image analysis program. To generate greater contrast in the microscope at interface 1, the video had to be recorded at $10 \times$ with a NIKON Labophot 2 illuminated-field microscope, and was recorded immediately after activation of the sperm cells.

\subsection{Video Capture Analysis with CASA Plug-In Application}

We used a 519CU 5.OM CMOS camcorder connected to a NIKON Labophot 2 microscope, which was connected to a Samsung computer with a $2.4 \mathrm{Ghz}$ Intel Core i5-2430 m processor, and the videos were recorded by using the Micrometrics LE program from the beginning of sperm cell activation to the end of their movement. The output video format was .AVI at 1280 by 968 pixels and 10 to 90 frames per second, which is the standard format for ImageJ. However, the video codec was converted from the output format, using the freeware program Any Video Converter, to get video images from the plug-in analysis, which is available at

http://www.any-video-converter.com. To transform the videos, the following changes were made in video options: "video codec", MPEG; "video size", original; "video bit rate", highest; and "video rate frames" identical to the following. Videos were imported into ImageJ and edited before analysis using the CASA plug-in. Editing involved inverting the video by choosing edit $>$ invert, which inverts the video colours. To obtain grayscale image, Image $>$ Type $>$ 8-bit grayscale was selected. To increase the contrast of the sperm cells, Image $>$ Adjust $>$ Brightness/ contrast (70/240) was used; this depends on the brightness of the video and the colour difference between the background and the sperm cells. To mark the sperm cells, Image $>$ Adjust $>$ Threshold (0/200) was used. A number sperm cells, between 10 at 150 single cells, was analyzed in the video. Threshold; mark a number of sperm in the video sample and select any single sperm cells for the track obtained, which leaves the sperm cells very accentuated and discernible. A summary of the protocol sequence is presented in Figure S1 and Table 1.

\subsection{Analysis Using SCA}

The samples were observed in a Nikon Eclipse E200 microscope with the phase filter on dark-field and a 10× objective; video images were acquired with a Basler sca78054tc camera at 10 frames per second. The samples were analyzed with SCA-CASA software (Microptic S.L. System Version 6, Barcelona, Spain), we used the follow pre-setting parameters, $10<$ area $<90 \mu^{2}$; VCL $10<$ slow $<45<$ medium $<100<$ faster $\mu \mathrm{m} /$ s; progressives $>80 \%$ of strength and circular $<50 \%$ linearity, modified for previous report [27]. The conditions were similar to those used for the experiment with the ImageJ CASA plug-in, and the temperature was controlled at $4^{\circ} \mathrm{C}$ : $10 \mu \mathrm{l}$ of semen were applied to a slide (no chamber is required for these CASA models), and quickly 
Table 1. Parameters changes used in the software.

\begin{tabular}{lccc}
\hline \multirow{2}{*}{ Parameters } & \multicolumn{3}{c}{ Software condition } \\
\cline { 2 - 4 } & Condition 1 & Condition 2 & Condition 3 \\
\hline Maximum sperm size (pixels) & 1000 & 1000 & 1000 \\
Minimum track length (frames) & 10 & 10 & 10 \\
Maximum sperm velocity between frames (pixels) & 30 & 60 & 90 \\
Minimum VCL for motility $(\mu \mathrm{m} / \mathrm{s})$ & 1 & 1 & 1 \\
Minimum VAP for motility $(\mu \mathrm{m} / \mathrm{s})$ & 1 & 1 & 1 \\
Minimum VSL for motility $(\mu \mathrm{m} / \mathrm{s})$ & 1 & 10 & 10 \\
Frame rate (frame per second) & 10 & 1 & 1 \\
\hline
\end{tabular}

Different parameters changes used in the software to obtain kinetic values from fish sperm samples. The frames were changed for different observations, but the other values were maintained.

mounted on the optical microscope. Once the sample was focused, $20 \mu \mathrm{l}$ of Powermilt $(1 \times)$ activation solution was applied and then coverslip of $18 \times 18 \mathrm{~mm}$ is used to finish the sample, three video recordings from five different fields were observed and the kinetic parameters was registered using the setting for commercial software.

\subsection{Statistical Analysis}

Unless indicated otherwise, all of the results, including the image analysis, are presented as mean \pm standard error of the mean (SEM). Analysis of variance (ANOVA) was performed to compare the effects of days in storage or Students-tests when comparing control and simple conditions. The samples were previously analysed for homogeneity by the D'Agostino and Pearson Test. All values passed the normality test with alpha of 0.05 . Post-test Bonferroni analysis was conducted for all-paired comparisons. A probability (p) less than 0.05 was considered statistically significant for ANOVA or Student tests. All data were analyzed with the statistical program Prism 5.

\section{Results}

\subsection{Initial Conditions and Recovery Protocols}

With software obtained via the Internet, the initial conditions must be accounted for in order to optimize conditions for analyzing recorded videos requires and to ensure sample reproducibility. Table 1 shows a scheme of user-modifiable values within the program, of which three conditions were changed so as to detect differences in real samples. Figure 1(a) shows track sample patterns analyzed under the three different conditions detailed in Table 1. Average values for VCL in each of the three conditions are shown in Figures 1(b)-(d) show the values for VSL and VAP. The results indicate that condition three, in which the number of frames analyzed was increased to 90 frames per second, yielded different values and presented the highest kinetic values of the three conditions. This evidence supports the use of this protocol to analyze samples. Upon obtaining the data, we proceeded to test the software by first observing and analyzing non-activated samples, after which the same samples were subsequently activated using an optimized protocol and then reassessed. Figure 2(a) illustrates the change in VCL from an inactivated to an activated state; a trace from an analyzed sample after activation is shown in the inset. The results indicate that the protocol makes possible the identification of samples possessing high motility after activation. Figure 2(b) and Figure 2(c) correspond to the analysis of the other two parameters, VSL and VAP. The data indicates that this protocol reveals important differences in the analysis and can differentiate samples that have been activated under laboratory conditions. We then compared the values of the kinetic parameters obtained with ImageJ and the CASA plug-in with those from a commercial CASA system (SCA, Microptic S.L.). Figure 2(d) shows the values for VSL: no statistically significant differences were observed. Similarly, no differences were observed in the values of the other parameters, as shown in Figure 2(e) and Figure 2(f). These results support the idea that this low-cost system can be used to observe kinetic parameters in salmon semen samples. 


\subsection{Motility Study and Changes over Time; Acute Observations}

To evaluate the sensitivity of the system optimized in our laboratory, the video recording of a sample was divided into three time periods and the kinetic values were evaluated for each period. Data were analyzed and, as shown in Figure 3(a), no differences in the VCL values could be observed at the different time periods. VSL values shown in Figure 3(b) do not show any differences between the observed time periods. The VAP values shown in Figure 3(c) not show change according to the time condition. Two new parameters that are of interest were calculated as well: the percentage motility and sample linearity. However as shown in Figure 3(d) and

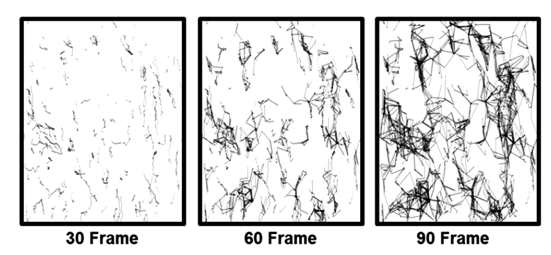

(a)

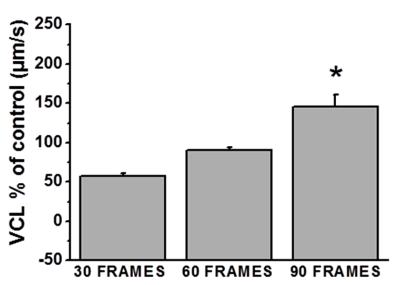

(b)

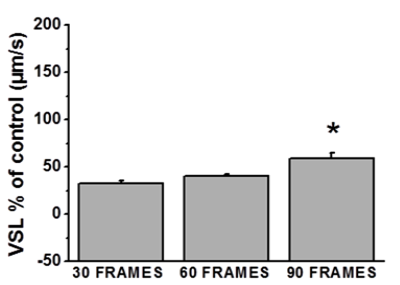

(c)

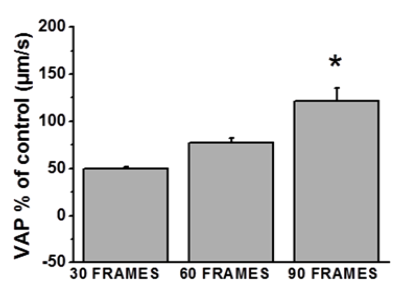

(d)

Figure 1. Parameter changes for kinetic observations. (a) Representative images for tracks observed at different frames for semen samples; (b) A bar graph of VCL values for activated semen with different frame conditions; (c) A bar graph of VSL observed with different frame conditions; (d) A bar graph of VAP values for activated semen observed with different frame conditions. Each bar (mean \pm SEM) represents measurements from at least five different experiments. The asterisks indicate $\mathrm{P}<0.05$ (one-way ANOVA).

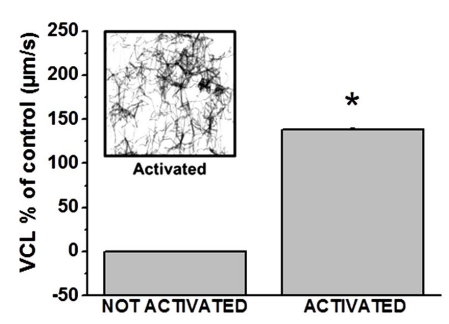

(a)

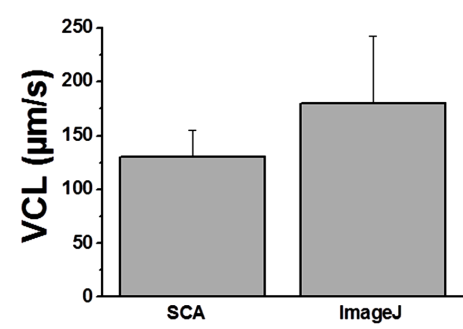

(d)

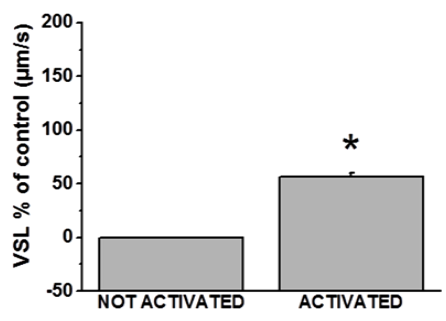

(b)

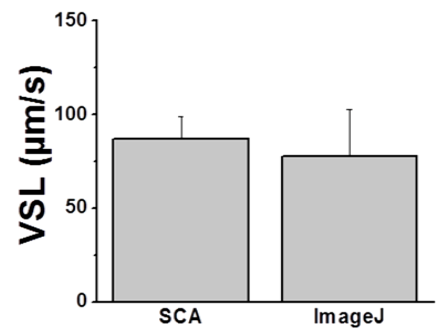

(e)

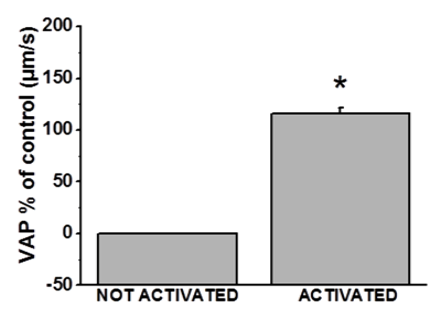

(c)

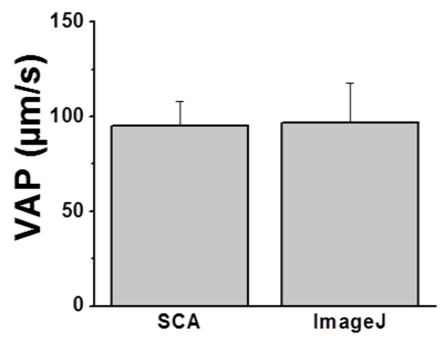

(f)

Figure 2. Parameter changes for kinetic observations in activated samples. (a) Bar graph of VCL values for activated semen. The inset is a representative image for a track observed for an activated semen sample; (b) A bar graph of VSL values for activated semen; (c) A bar graph of VAP values for activated semen; (d) A bar graph comparing the VCL value from SCA vs. ImageJ; (e) A bar graph comparing VSL value from SCA vs. ImageJ (f) A bar graph comparing VAP value from SCA vs. ImageJ. Each bar (mean $\pm \mathrm{SEM}$ ) represents measurements from at least five different experiments. The asterisks indicate $\mathrm{P}<$ 0.05 (Student's t test). 


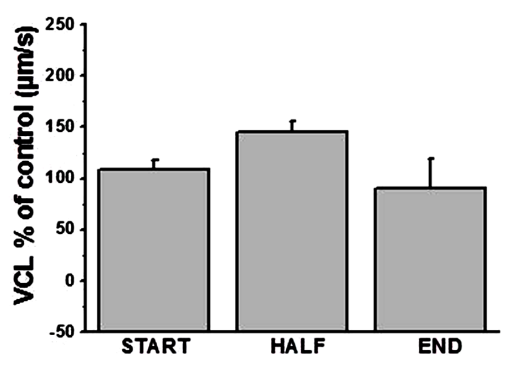

(a)

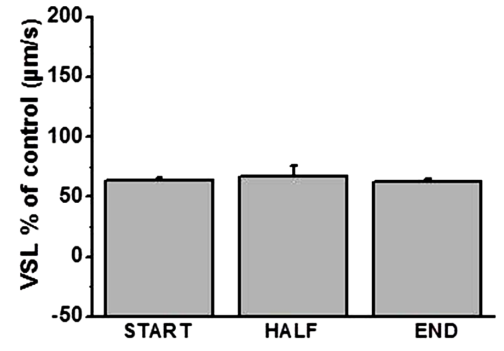

(b)

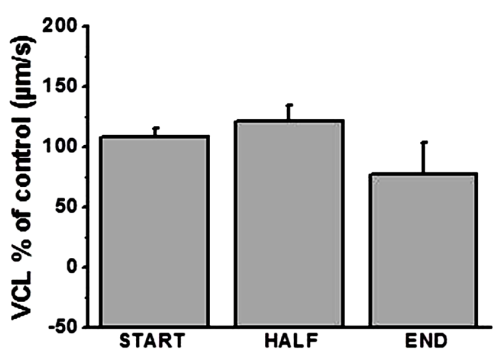

(c)

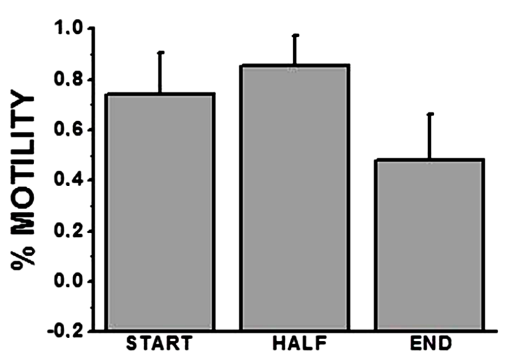

(d)

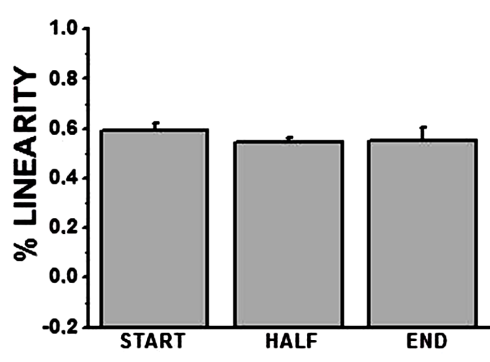

(e)

Figure 3. Parameter changes at different times. (a) Bar graph of VCL values for activated semen observed at different time points; (b) Bar graph of VSL values for activated semen observed at different time points; (c) A bar graph of VAP values for activated semen different time points; (d) Bar graph of activated semen motility values at different time points; (e) A bar graph of activated semen linearity values at different time points. Each bar (mean \pm SEM) represents measurements from at least five different experiments. Data do not show significant difference.

Figure 3(e), no significant differences were observed. These results indicate that although this protocol is able to analyse motile samples, it is not sensitive enough to identify potential small changes when observing the same sample in acute observation.

\subsection{Motility Study and Variation over Time; Periodic Observations}

Semen samples from healthy Salmo salar were collected using the standard protocol; the kinetic values were registered and semen extractions were repeated on different days. Figure 4(a) shows examples of traces registered from samples upon initial observation and after 10 and 15 days of storage in the laboratory according to conditions of oxygenation, temperature and movement. Figures 4(b)-(d) show the VCL, VSL and VAP values on different days and illustrate the significant reduction in these values that occurs when samples are preserved in low-temperature and shaken every day for oxygenation, as previous described by [25]; the kinetic properties were quantified using an optimized protocol in our laboratory. The data indicate that this easy way for the analysis of salmon sperm cell kinetics is able to distinguish changes that occur during storage and can be used to assess sperm cell quality. Interestingly, when retrieving values published by other groups and comparing these values with our own data, as shown in Table 2, we did not find any significant differences, suggesting that our values are within the expected range for a healthy sample and can detect variations in the kinetics of a sample.

\section{Discussion}

We used the CASA plug-in application, which was developed for use with open source software, such as ImageJ. Use of these methods can reduce the cost associated with measuring the kinetic parameters. We analyzed fish semen, in particular Salmon salar sperm cells because they have a natural particularity of not being motile before they are activated. This natural occurrence helped us to manipulate the sample in the laboratory and introduces a new protocol to the aquaculture industry for evaluating the quality of sperm cells. With this protocol, observable differences are shown, and previous observations can be compared with similar models [19]. Furthermore, in the last year, several publications include the methodology for obtaining kinetic data from fish semen with the ImageJ-CASA system [20] [21]. Part of these developments is marked by the use of automatic 

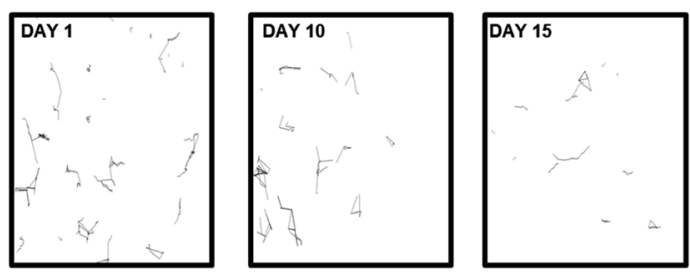

(a)

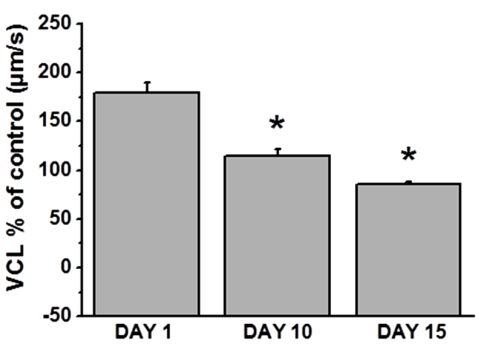

(b)

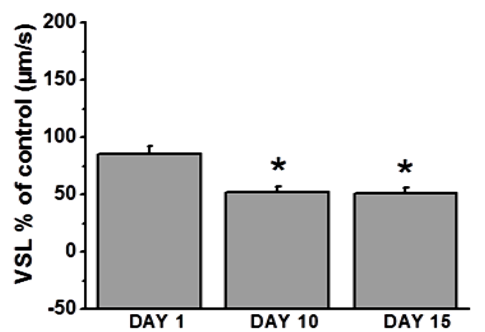

(c)

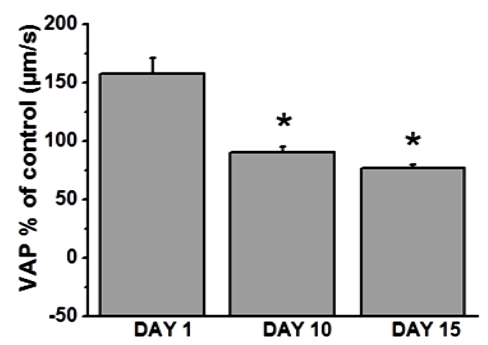

(d)

Figure 4. Parameter changes at different storage days. (a) Representative images for a track observed at different storage days. (b) A bar graph of VCL values for activated semen at the initial time and after the indicated number of days of storage (c) A bar graph of VSL values for activated semen at the initial time and after the indicated number of days of storage; (d) A bar graph of VAP values for activated semen at the initial time and after the indicated number of days of storage. Each bar (mean \pm SEM) represents measurements from at least five different experiments. The asterisks indicate P $<0.05$ (ANOVA).

Table 2. Kinetics values described.

\begin{tabular}{|c|c|c|c|c|c|}
\hline \multirow{2}{*}{ Author } & \multicolumn{5}{|c|}{ Kinetics parameter } \\
\hline & $\%$ motile & VCL $(\mu \mathrm{m} / \mathrm{s})$ & VAP $(\mu \mathrm{m} / \mathrm{s})$ & VSL $(\mu \mathrm{m} / \mathrm{s})$ & Species \\
\hline Present study & $68 \pm 4$ & $180 \pm 63$ & $78 \pm 27$ & $97 \pm 26$ & Salmo salar \\
\hline $\begin{array}{l}\text { Dziewulska, A. Rzemieniecki and } \\
\text { J. Domagala, } 2011\end{array}$ & & $178.4 \pm 63.6$ & $155.7 \pm 58.6$ & $114.8 \pm 72.9$ & Salmo salar \\
\hline Lahnsteiner F. et al., 2005 & & $91.4 \pm 20.8$ & & & Onchorhynchus mykiss \\
\hline $\begin{array}{l}\text { Wilson-Leedy J. and } \\
\text { Ingermann R., } 2007\end{array}$ & $90 \pm 6$ & $104 \pm 9$ & $77 \pm 15$ & $66 \pm 17$ & Danio rerio \\
\hline
\end{tabular}

Comparison of kinetics values described by other researchers for similar species with our results.

systems to describe the quality of the semen samples (for example, analysis of the percentage of chromocenter in the sperm cell nucleus), and make a correlation with quality and motility of semen sample [3] or in human spermatozoa for study flow cytometry [2].

Traditionally, semen quality is assessed through the direct observation of the sample or by using the kinetics of sperm cells as a method of quantifying semen quality, particularly sperm cell motility. This quantification is typically performed through subjective observation by a trained observer or with the aid of programs, such as CASA [22]. However, these programs are expensive and require specialized instruments, therefore, their use is limited. Salmon sperm cells can be activated under laboratory conditions; the duration of motility can be assessed to evaluate sperm cell quality, the observation of initial motility, and the loss of certain properties as sperm cells in the sample lose their motility [15] [25]. Measuring semen quality is crucial in order to use these cells more efficiently. The success of conservation and artificial reproduction efforts depends on semen quality [18]. The software used in these experiments has been updated in our laboratory by analysing videos obtained via conventional illuminated-field microscopes with a 5-megapixel camera. This equipment is easily accessible, and the software is free. Figure S1 presents a step-by-step schematic of the protocol, including a checklist for video acquisition and the parameters that are measured by the ImageJ CASA plug-in, which we used for fish semen. However, this system can be adapted for other sample types and updated in any laboratory [21]. Although the protocol may appear complex, it is not difficult to follow, reduces the number of the steps, the costs 
are low, and only the conditions for different species needs to be checked and accordingly adjusted. We modified the initial parameters to generate reproducible observations that correspond with published values and detect differences between the samples. We described one important step for the proper acquisition of frames for video capture [21]. Previous reports helped to us to introduce proper modifications to the plug-in; the frame rate is described as a critical parameter for obtaining accurate recordings for kinetic analyses [20]. After modifying the protocol, we obtained differing values when the samples were activated using Powermilt.

With this test system, our protocol was able to identify activated sperm cells where the activated condition yields different values for VCL, VSL and VAP. When evaluating the sensitivity of our protocol to variations within a sample, we found that it was not possible to identify significant changes in the parameters examined (e.g., VCL, VSL and VAP). Furthermore, when compared with values obtained from the SCA system, no differences were detected, suggesting that the ImageJ CASA system is comparable to a conventional CASA system. The values observed under initial conditions of normal semen from Atlantic salmon (Salmo salar) showed similar values to those reported for similar species [19] [24] [28], suggesting that our system using open source software provides reproducible and comparable results, supporting the idea that identical kinetic parameters can be produced with this low-cost system. However, the SCA software can show different values in the semen sample and can detect variations in short time periods. We added other parameters or conditions to the ImageJ CASA system; however, these were insufficient for indicating changes in the samples. The previous results indicate that our protocol can be used to quantify changes in semen samples, but small variations in kinetics cannot be detected, limiting its use in certain processes. The storage of semen samples for several days is a common practice in the industry, where samples are often collected, cryopreserved and then observed upon activation. It is important to evaluate the quality of semen samples as well as their kinetics [18] [29]. To assess sperm quality with our system, we studied the effect of storage over time. VCL, VSL and VAP were used to identify decreases in the kinetics (and therefore the quality of semen over several days of storage) and reduced the time of activation, previously described as one indication of quality reduction [15] [25].

\section{Conclusion}

Our data indicate that a low-cost system, ImageJ CASA, enables basic observation of the kinetics of salmon sperm cells, with some limitations, such as the sensitivity of the system to acute changes, although VSL, VCL and VAP can be detected and provide similar values to other CASA systems and to previous studies [28] [30]. We suggest the use of these models for low-cost protocol for kinetic observation in other species (bovine, human, caprine, porcine and others). The protocol has limits and needs a test sample before it is implemented for other species, but it is a viable alternative for generating new data in more semen models.

\section{Acknowledgements}

Jorge Parodi is supported by a MECESUP-UCT 0804 grant. Alfredo Ramírez-Reveco is supported by a FONDEFD08I1076. We are indebted to Professor Ian Scott for his translation, revision and editing. Language editing services were provided by www.journalrevisions.com, with support from a DGIP-UCT Fellowship. We thank Dr. Valdebenito for the semen sample and powermilt solution.

\section{References}

[1] Parodi, J. (2014) Motility, Viability, and Calcium in the Sperm Cells. Systems Biology in Reproductive Medicine, 60, 65-71. http://dx.doi.org/10.3109/19396368.2013.869273

[2] Buckman, C., George, T.C., Friend, S., Sutovsky, M., Miranda-Vizuete, A., Ozanon, C., Morrissey, P. and Sutovsky, P. (2009) High Throughput, Parallel Imaging and Biomarker Quantification of Human Spermatozoa by Image Stream Flow Cytometry. Systems Biology in Reproductive Medicine, 55, 244-251. http://dx.doi.org/10.3109/19396360903056224

[3] Alladin, N., Moskovtsev, S.I., Russell, H., Kenigsberg, S., Lulat, A.G.-M. and Librach, C.L. (2013) The Three-Dimensional Image Analysis of the Chromocenter in Motile and Immotile Human Sperm. Systems Biology in Reproductive Medicine, 59, 146-152. http://dx.doi.org/10.3109/19396368.2013.772679

[4] Butts, I.A., Babiak, I., Ciereszko, A., Litvak, M.K., Slowinska, M., Soler, C. and Trippel, E.A. (2011) Semen Characteristics and Their Ability to Predict Sperm Cryopreservation Potential of Atlantic Cod, Gadus morhua L. Theriogenology, 75, 1290-1300. http://dx.doi.org/10.1016/j.theriogenology.2010.11.044 
[5] Ding, F., Lall, S.P., Li, J., Lei, J., Rommens, M. and Milley, J.E. (2011) Cryopreservation of Sperm from Atlantic Halibut (Hippoglossus hippoglossus L.) for Commercial Application. Cryobiology, 63, 56-60. http://dx.doi.org/10.1016/j.cryobiol.2011.04.009

[6] Viveiros, A.T. and Godinho, H.P. (2009) Sperm Quality and Cryopreservation of Brazilian Freshwater Fish Species: A Review. Fish Physiology and Biochemistry, 35, 137-150. http://dx.doi.org/10.1007/s10695-008-9240-3

[7] Sanchez-Rodriguez, M. and Billard, R. (1977) Conservation de la motilité et du pouvoir fécondant du sperme de truite arc-en-ciel maintenu à des températures voisines de $0^{\circ} \mathrm{C}$. Bulletin Francais De La Peche Et De La Pisciculture, 265, 143-152. http://dx.doi.org/10.1051/kmae:1977009

[8] Bobe, J. and Labbe, C. (2009) Egg and Sperm Quality in Fish. General and Comparative Endocrinology, 165, 535548. http://dx.doi.org/10.1016/j.ygcen.2009.02.011

[9] Maldjian, A., Pizzi, F., Gliozzi, T., Cerolini, S., Penny, P. and Noble, R. (2005) Changes in Sperm Quality and Lipid Composition during Cryopreservation of Boar Semen. Theriogenology, 63, 411-421. http://dx.doi.org/10.1016/j.theriogenology.2004.09.021

[10] Viveiros, A.T., Orfao, L.H., Nascimento, A.F., Correa, F.M. and Caneppele, D. (2012) Effects of Extenders, Cryoprotectants and Freezing Methods on Sperm Quality of the Threatened Brazilian Freshwater Fish Pirapitinga-do-Sul Brycon opalinus (Characiformes). Theriogenology, 78, 361-368. http://dx.doi.org/10.1016/j.theriogenology.2012.02.015

[11] Beirao, J., Soares, F., Herraez, M.P., Dinis, M.T. and Cabrita, E. (2009) Sperm Quality Evaluation in Solea senegalensis during the Reproductive Season at Cellular Level. Theriogenology, 72, 1251-1261. http://dx.doi.org/10.1016/j.theriogenology.2009.07.021

[12] Kowalski, R.K., Hliwa, P., Cejko, B.I., Krol, J., Stabinski, R. and Ciereszko, A. (2012) Quality and Quantity of Smelt (Osmerus eperlanus L.) Sperm in Relation to Time after Hormonal Stimulation. Reproductive Biology, 12, 231-246. http://dx.doi.org/10.1016/S1642-431X(12)60088-6

[13] Alavi, S.M. and Cosson, J. (2005) Sperm Motility in Fishes. I. Effects of Temperature and pH: A Review. Cell Biology International, 29, 101-110. http://dx.doi.org/10.1016/j.cellbi.2004.11.021

[14] Alavi, S.M., Cosson, J., Karami, M., Amiri, B.M. and Akhoundzadeh, M.A. (2004) Spermatozoa Motility in the Persian sturgeon, Acipenser persicus: Effects of pH, Dilution Rate, Ions and Osmolality. Reproduction, 128, 819-828. http://dx.doi.org/10.1530/rep.1.00244

[15] Redondo-Muller, C., Cosson, M.P., Cosson, J. and Billard, R. (1991) In Vitro Maturation of the Potential for Movement of Carp Spermatozoa. Molecular Reproduction and Development, 29, 259-270. http://dx.doi.org/10.1002/mrd.1080290308

[16] Vladic, T.V. and Jarvi, T. (2001) Sperm Quality in the Alternative Reproductive Tactics of Atlantic salmon: The Importance of the Loaded Raffle Mechanism. Proceedings of the Royal Society B: Biological Sciences, 268, $2375-2381$. http://dx.doi.org/10.1098/rspb.2001.1768

[17] Nyina-Wamwiza, L., Milla, S., Pierrard, M.A., Rurangwa, E., Mandiki, S.N., Van Look, K.J. and Kestemont, P. (2011) Partial and Total Fish Meal Replacement by Agricultural Products in the Diets Improve Sperm Quality in African catfish (Clarias gariepinus). Theriogenology, 77, 184-194. http://dx.doi.org/10.1016/j.theriogenology.2011.07.032

[18] Viveiros, A.T., Isau, Z.A., Caneppele, D. and Leal, M.C. (2012) Sperm Cryopreservation Affects Postthaw Motility, but Not Embryogenesis or Larval Growth in the Brazilian Fish Brycon insignis (Characiformes). Theriogenology, 78, 803-810. http://dx.doi.org/10.1016/j.theriogenology.2012.03.028

[19] Wilson-Leedy, J.G. and Ingermann, R.L. (2007) Development of a Novel CASA System Based on Open Source Software for Characterization of Zebrafish Sperm Motility Parameters. Theriogenology, 67, 661-672. http://dx.doi.org/10.1016/j.theriogenology.2006.10.003

[20] Purchase, C.F. and Earle, P.T. (2012) Modifications to the Imagej Computer Assisted Sperm Analysis Plugin Greatly Improve Efficiency and Fundamentally Alter the Scope of Attainable Data. Journal of Applied Ichthyology, 28, 10131016. http://dx.doi.org/10.1111/jai.12070

[21] Sanches, E.A., Marcos, R.M., Okawara, R.Y., Caneppele, D., Bombardelli, R.A. and Romagosa, E. (2013) Sperm Motility Parameters for Steindachneridion parahybae Based on Open-Source Software. Journal of Applied Ichthyology, 29, 1114-1122. http://dx.doi.org/10.1111/jai.12165

[22] Rurangwa, E., Volckaert, F.A., Huyskens, G., Kime, D.E. and Ollevier, F. (2001) Quality Control of Refrigerated and Cryopreserved Semen Using Computer-Assisted Sperm Analysis (CASA), Viable Staining and Standardized Fertilization in African Catfish (Clarias gariepinus). Theriogenology, 55, 751-769. http://dx.doi.org/10.1016/S0093-691X(01)00441-1

[23] Butts, I.A., Ward, M.A., Litvak, M.K., Pitcher, T.E., Alavi, S.M., Trippel, E.A. and Rideout, R.M. (2011) Automated Sperm Head Morphology Analyzer for Open-Source Software. Theriogenology, 76, 1756-1761. http://dx.doi.org/10.1016/j.theriogenology.2011.06.019 
[24] Lahnsteiner, F., Berger, B., Horvath, A., Urbanyi, B. and Weismann, T. (2000) Cryopreservation of Spermatozoa in Cyprinid Fishes. Theriogenology, 54, 1477-1498. http://dx.doi.org/10.1016/S0093-691X(00)00469-6

[25] Ubilla, A. and Valdebenito, I.N. (2011) Use of Antioxidants on Rainbow Trout Oncorhynchus mykiss (Walbaum, 1792) Sperm Diluent: Effects on Motility and Fertilizing Capability. Latin American Journal of Aquatic Research, 39, 338343. http://dx.doi.org/10.3856/vol39-issue2-fulltext-15

[26] Figueroa, E., Risopatron, J., Sanchez, R., Isachenko, E., Merino, O., Isachenko, V. and Valdebenito, I. (2013) Spermatozoa Vitrification of Sex-Reversed Rainbow Trout (Oncorhynchus mykiss): Effect of Seminal Plasma on Physiological Parameters. Aquaculture, 372, 119-126. http://dx.doi.org/10.1016/j.aquaculture.2012.10.019

[27] Ramirez, A.R., Castro, M.A., Angulo, C., Ramio, L., Rivera, M.M., Torres, M., Rigau, T., Rodriguez-Gil, J.E. and Concha, I.I. (2009) The Presence and Function of Dopamine Type 2 Receptors in Boar Sperm: A Possible Role for Dopamine in Viability, Capacitation, and Modulation of Sperm Motility. Biology of Reproduction, 80, 753-761. http://dx.doi.org/10.1095/biolreprod.108.070961

[28] Dziewulska, K., Rzemieniecki, A., Czerniawski, R. and Domagala, J. (2011) Post-Thawed Motility and Fertility from Atlantic Salmon (Salmo salar L.) Sperm Frozen with Four Cryodiluents in Straws or Pellets. Theriogenology, 76, $300-$ 311. http://dx.doi.org/10.1016/j.theriogenology.2011.02.007

[29] Valdebenito, I.I., Gallegos, P.C. and Effer, B.R. (2013) Gamete Quality in Fish: Evaluation Parameters and Determining Factors. Zygote, 1-21. http://dx.doi.org/10.1017/S0967199413000506

[30] Lahnsteiner, F., Berger, B., Grubinger, F. and Weismann, T. (2005) The Effect of 4-Nonylphenol on Semen Quality, Viability of Gametes, Fertilization Success, and Embryo and Larvae Survival in Rainbow Trout (Oncorhynchus mykiss). Aquatic Toxicology, 71, 297-306. http://dx.doi.org/10.1016/j.aquatox.2004.11.007

\section{Supplementary}

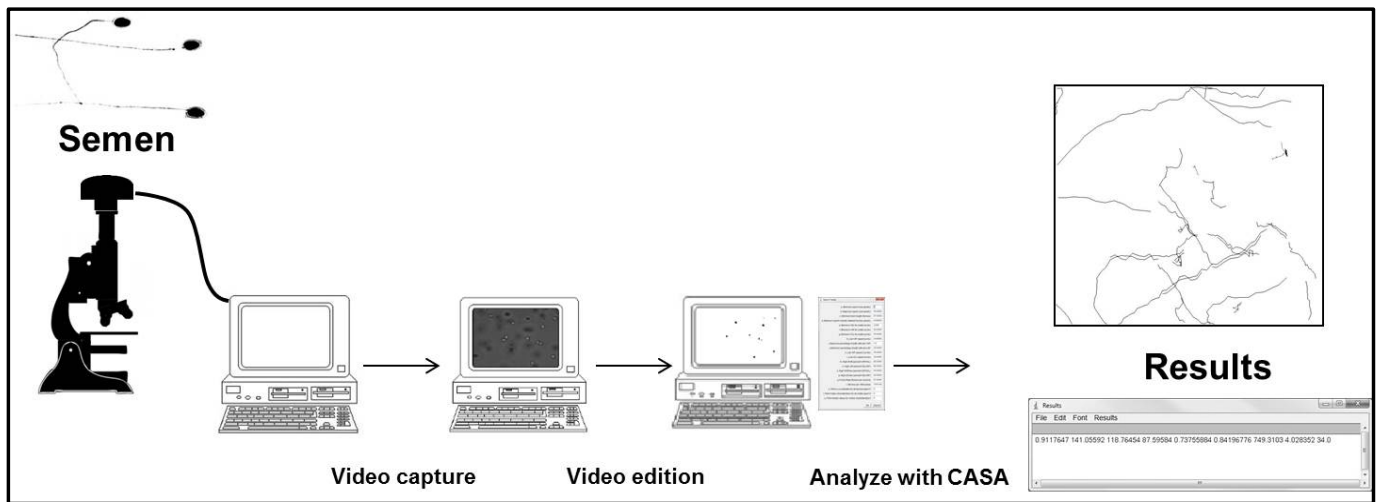

Figure S1. Presenting the method used to obtain and analyze fish sperm kinetics The diagram shows the different steps used in the protocol to generate kinetic information from semen samples. 
Scientific Research Publishing (SCIRP) is one of the largest Open Access journal publishers. It is currently publishing more than 200 open access, online, peer-reviewed journals covering a wide range of academic disciplines. SCIRP serves the worldwide academic communities and contributes to the progress and application of science with its publication.

Other selected journals from SCIRP are listed as below. Submit your manuscript to us via either submit@scirp.org or Online Submission Portal.
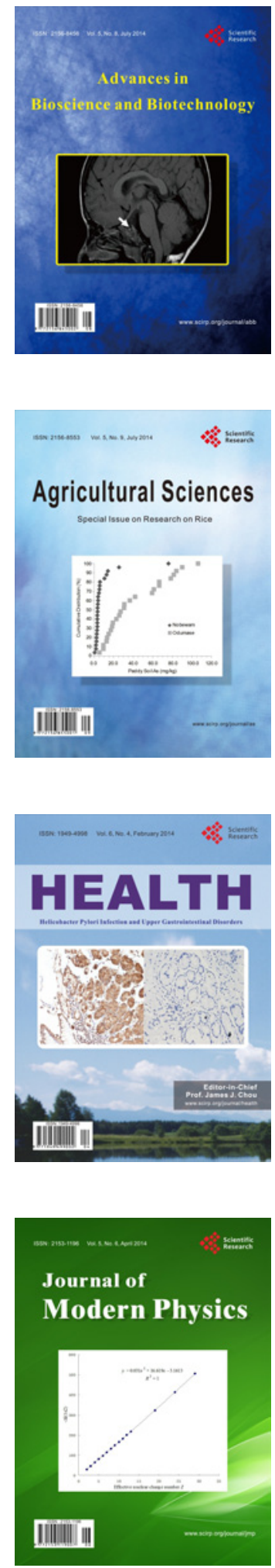
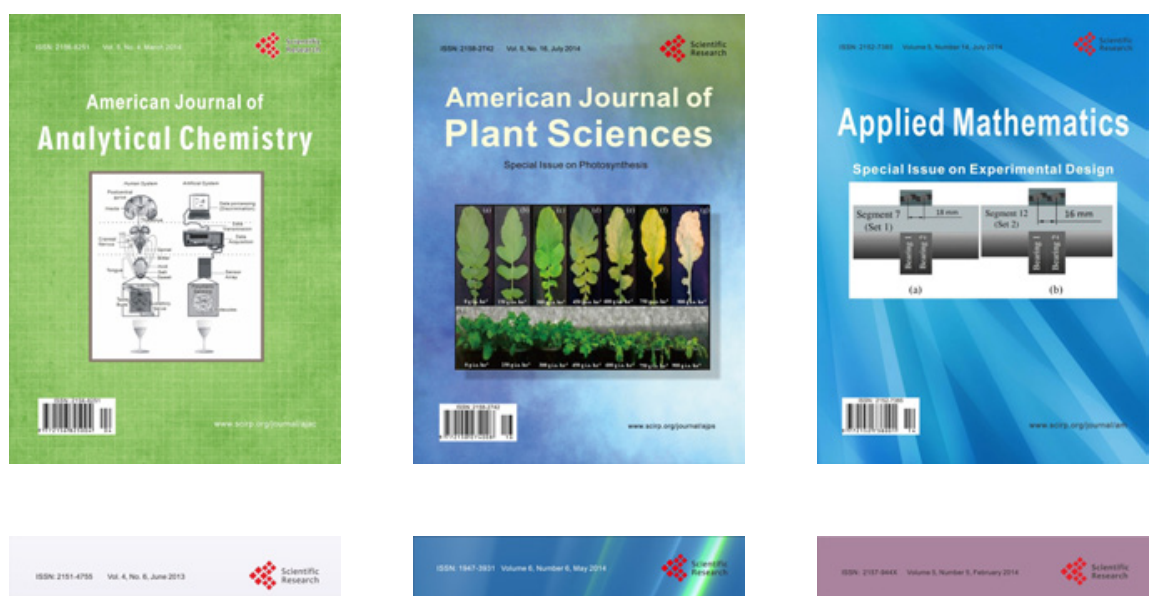

Creative Education
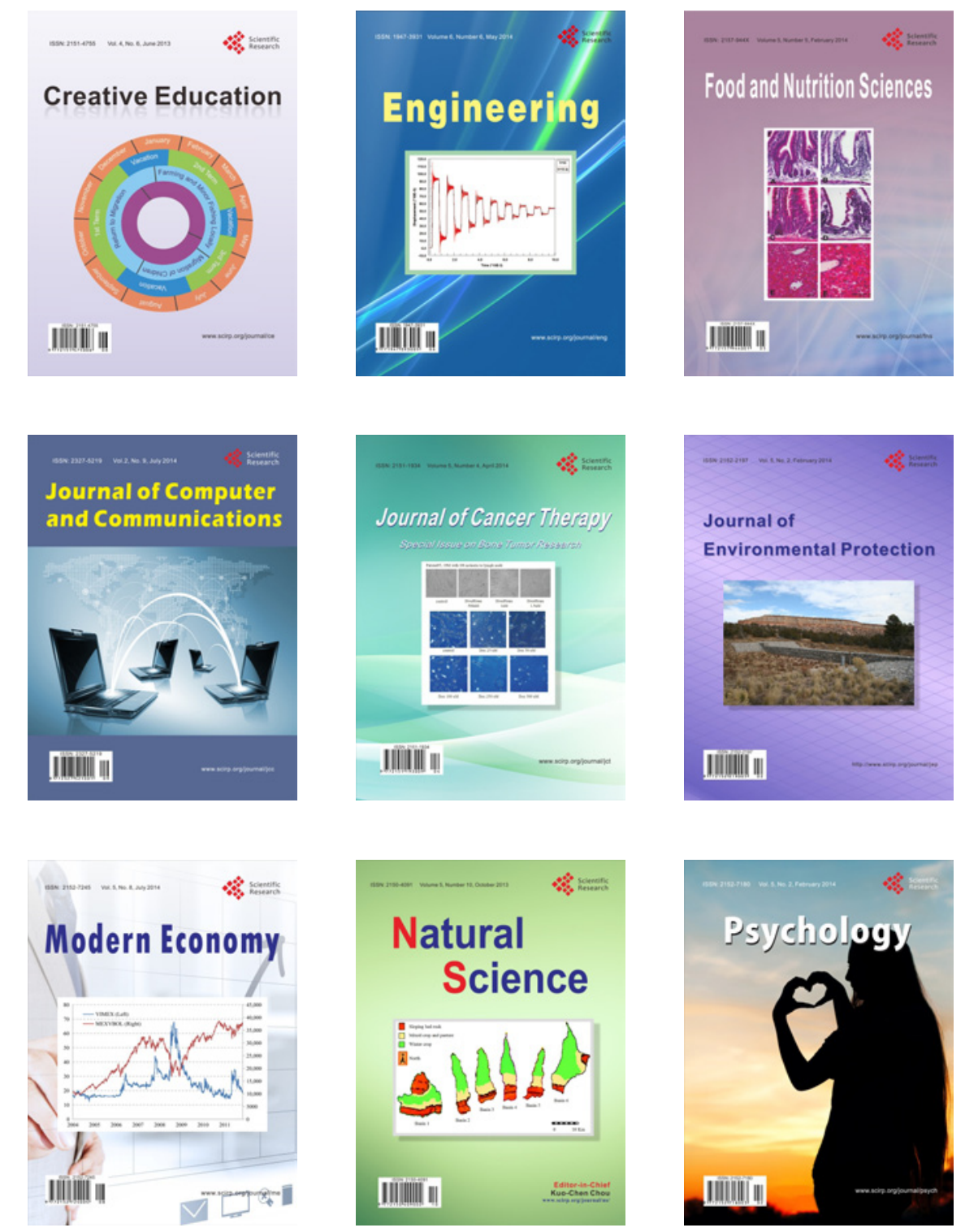